\title{
Funçóes Executivas na Dislexia do Desenvolvimento: Revendo EVIdênCIAS De Pesquisas ${ }^{1}$
}

\author{
EXECUTIVE FunCTION IN DEVELOPMENTAL DySLEXIA: RESEARCH EVIDENCE REVIEW
}

\author{
Giovanna Beatriz Kalva MEDINA² \\ Maria de Fátima Joaquim MINETTO3 \\ Sandra Regina Kirchner GUIMARÁES ${ }^{4}$
}

\begin{abstract}
RESUMO: o objetivo deste artigo é fazer uma revisão sistemática de literatura a fim de analisar produções científicas que abordam as funçôes executivas (FE) e a dislexia. O método consiste num levantamento de artigos publicados na Biblioteca Virtual em Saúde, no Portal de Periódicos da CAPES e na PUBMED. Foram selecionados 28 trabalhos, sendo 9 do PUBMED, 14 dos Periódicos da CAPES, 4 da BVS e um manualmente. Resultados indicaram que os estudos selecionados não avaliam as FE da mesma forma. Alguns avaliam as FE como um componente global, o qual demonstra pouca diferença comparando os disléxicos a leitores típicos, outros avaliam os componentes principais das FE, que são o Controle Inibitório, a Flexibilidade Cognitiva e a Memória de Trabalho (MT), e um terceiro grupo de estudos avalia a MT também em seus subcomponentes: MT visuoespacial, executivo central e MT verbal ou fonológica. É possível concluir que o estudo das FE em disléxicos está em pleno desenvolvimento. Nos últimos 5 anos, o interesse nesta temática aumentou, inclusive no Brasil, expresso pelo aumento no número de estudos publicados. Porém, ainda há muito que se conhecer, principalmente sobre o efeito de intervençóes envolvendo as FE.
\end{abstract}

PALAVRAS-CHAVE: Educação Especial. Funçōes Executivas. Dislexia. Leitura.

\begin{abstract}
The objective of this article is to make a systematic review of literature in order to analyze scientific productions that deal with executive functions (EF) and dyslexia. The method consists on a selection of articles published in the Virtual Health Library (BVS), in the CAPES Periodicals and PUBMED. Were selected 28 papers, 9 from PUBMED, 14 from CAPES Periodicals, 4 from the BVS and one manually. Results indicated that the selected studies do not evaluate FE in the same way. Some evaluate EFs as a global component, which shows little difference comparing dyslexics to typical readers, others assess the main components of EFs, which are Inhibitory Control, Cognitive Flexibility, and Working Memory (WM), and a third group of studies evaluates WM also in its subcomponents: visuo-spatial WM, central executive and verbal or phonological WM. It is possible conclue that the study of EF in dyslexics is in full development. In the last 5 years, interest in this subject has increased, including in Brazil, expressed by the increase in the number of published studies, but there is still a lot to know, especially about the effect of interventions involving EF.
\end{abstract}

KEYWORD: Special Education. Executive Function. Dyslexia. Reading.

\section{INTRODUÇão}

A leitura é uma atividade neurocognitiva de alta complexidade que requer o reconhecimento das letras e suas combinaçóes para que sejam convertidos nos devidos sons (decodificação grafema-fonema) (ELLIS, 1995). A dislexia do desenvolvimento, também nominada como um distúrbio do reconhecimento de palavras, ou da habilidade empobrecida da leitura de palavras, apresenta bases neurológicas que afetam a alfabetização no nível do reconhecimen-

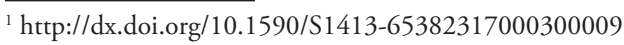

${ }^{2}$ Docente no curso de Psicologia do Centro Universitário FAE, Curitiba, PR, Brasil. giovannabkmedina@gmail.com.

${ }^{3}$ Professora do Departamento de Teoria e Fundamentos da Educação da Universidade Federal do Paraná e do Programa de Pós-Graduação da Universidade Federal do Paraná, Curitiba, PR, Brasil. fa.minetto@gmail.com

${ }^{4}$ Professora Permanente do Programa de Pós-graduação em Educaçâo da Universidade Federal do Paraná. Líder do Grupo de Pesquisa Cognição, Aprendizagem e Desenvolvimento Humano. Curitiba, PR, Brasil. srkguimaraes@uol.com.br
} 
to (decodificação), da fluência e da compreeensão (HELLAND; ASBJØRNSEN, 2000; APA, 2014). Esse transtorno específico da aprendizagem da leitura afeta não somente habilidades envolvendo a linguagem, mas outras como a coordenação motora, a orientação temporal e espacial, que são fundamentadas pelas diferentes teorias da dislexia (CAIN, 2010), pois sabe-se que esta sintomatologia variada não é explicada por uma única variável (ALVES et al., 2011).

Muitas pesquisas têm sido desenvolvidas com o objetivo de identificar as funçóes cognitivas que podem estar em defasagem na pessoa que apresenta dislexia (SNOWLING, 2004; VELLUTINO; FLETCHER, 2013), e atualmente as pesquisas vêm direcionando o olhar para algumas áreas específicas, como as funçóes executivas (FE).

Para Gilbert e Burgess (2008), as FE envolvem os processos cognitivos de mais alto nível, que permitem ao indivíduo encontrar alternativas sobre o que fazer quando não sabe o que fazer e adaptando-se a circunstâncias desconhecidas. Lezak (1982, p.281) descreve as FE como o centro do desenvolvimento pessoal que está envolvido integralmente ou parcialmente em tudo que fazemos. É a capacidade necessária para elaborar objetivos, planejar como executá-los, acompanhando-os para que se desenvolvam de forma eficiente.

Destaca-se que FE é um termo guarda-chuva usado para se referir aos processos cognitivos e metacognitivos de mais alto nível de controle e gerenciamento de outros processos mentais, emocionais e comportamentais, tais como: planejamento, organizaçáo, autorregulação, monitoramento, intenção, tomada de decisão, categorização, resolução de problemas, motivação, atenção, concentração, adaptação à mudança, consciência de si, criatividade, dentre outras (LEZAK, 1982; GILBERT; BURGESS, 2008; CORSO et al., 2013; DIAMOND, 2013; MALLOY-DINIZ et al., 2014; SEABRA et al., 2014; PUREZA et al., 2015).

A descrição detalhada de cada componente das FE foi feita por Diamond (2013) a partir de uma revisão do estudo de Miyake et al. (2000), iniciando pela inibição ou controle inibitório (CI), que atua no controle do pensamento, da atenção, das emoçóes e do comportamento em direção à ação mais adequada em determinada situação. Por inibir estímulos irrelevantes ou pouco importantes em um determinado momento e para manter o foco, a inibição tem a função de atenção seletiva chamada de controle inibitório da atenção (SEABRA et al., 2014; DIAMOND, 2013). Na leitura, o controle inibitório tem um importante papel, pois o indivíduo precisa excluir estímulos distratores como sons do ambiente, realizar um movimento sequenciado da esquerda para a direita, com os olhos, processando as letras, palavras e frases, assim como se concentrando no conteúdo e inibindo outros pensamentos (LIMA, TRAVAINI, CIASCA, 2009). Esse controle acrescido dos demais componentes executivos e funçóes cognitivas permite a decodificação e a compreensão do texto.

Outro componente das FE é a memória de trabalho (MT) ou também chamada de memória operacional ou atualização (updating), definida como um sistema de armazenamento com capacidade limitada, que mantém e processa informaçóes verbais e visuoespaciais (não verbais), com o objetivo de dar apoio aos processos cognitivos, promovendo uma interface entre percepção, memória a longo prazo e ação. A MT é um modelo de multicomponentes com detalhes específicos que envolvem a alça fonológica, prancha visuoespacial (relacionados à memória a curto prazo), o executivo central (sistema supervisor responsável por manipular a informação armazenada nos componentes de apoio), e o buffer episódico (onde se armazenam 
as funçôes de várias procedências) (BADDELEY, 2003; SEABRA et al., 2014). A memória de trabalho na leitura é necessária para manter detalhes de uma história na mente, para construir uma representação coerente que dê sentido ao texto, em um contínuo, incluindo detalhes descobertos durante o processo (CARTWRIGHT, 2015).

A MT e o CI são, de alguma forma, as bases para a flexibilidade cognitiva (FC), o componente das FE, que envolve o pensar de forma flexível, a mudança de atitude ou ação diante de um novo elemento, ou ainda a abertura para novas possibilidades. Na leitura, verifica-se a atuação da flexibilidade cognitiva quando se lê as palavras respeitando-se o som de cada letra, mas também quando, na leitura de um texto, se respeita os sinais de pontuação, que marcam as pausas, a melodia e a entonação. Destaca-se que a leitura realizada desta forma é que expressa a compreensão leitora.

Diferentes autores (CORSO et al., 2013; BOVO et al., 2016) apontam que o desenvolvimento das habilidades cognitivas e metacognitivas - por exemplo: planejamento, monitoramento e controle inibitório - são essenciais no processo de aprendizagem e que déficits neste desenvolvimento estão presentes nas dificuldades de aprendizagem. Assim, infere-se que o desenvolvimento das funçóes executivas também impacta a aquisição da leitura dos disléxicos do desenvolvimento e esta é uma questão que precisa ser elucidada. Acredita-se que esclarecer as relaçóes entre as funções executivas e a aprendizagem inicial e/ou aperfeiçoamento da leitura instrumentaliza tanto os professores como outros profissionais que trabalham com estudantes disléxicos, a propor estratégias para o desenvolvimento destas funçôes e, consequentemente, melhorar o desempenho em leitura.

É neste contexto teórico que a presente revisão se insere, com o objetivo de analisar as produçóes científicas que abordam as funçóes executivas e a dislexia, verificando: quais os instrumentos mais utilizados para avaliar as funções executivas; como se desempenham os disléxicos em testes que avaliam as funçóes executivas; se as funçóes executivas são preditores da dislexia, qual é o papel das funções executivas na leitura de pessoas com dislexia e qual a contribuição que as funçóes executivas podem oferecer para a remediação da dificuldade de leitura (dislexia).

\section{Método}

A presente revisão sistemática de literatura foi desenvolvida a partir de um levantamento de artigos realizado na Biblioteca Virtual em Saúde (BVS), no Portal de Periódicos da CAPES e na Biblioteca Nacional de Medicina dos Estados Unidos da América (PUBMED). A pesquisa aconteceu no período de abril a junho de 2016, utilizando o descritor funçóes executivas adicionado dos descritores dislexia ou dificuldade de leitura ou transtorno de leitura ou distúrbio de leitura, nos idiomas português, inglês e espanhol.

Os critérios para inclusão foram: trabalhos empíricos envolvendo os descritores escolhidos; pesquisas envolvendo crianças, jovens ou adultos; estudos de diferentes origens desde que publicados em português, inglês ou espanhol; abordaram uma ou mais FE relacionando a leitura em pessoas com dificuldade/transtorno/distúrbio na leitura ou dislexia. Foram excluídos os trabalhos que: não abordavam a temática das FE relacionada à dislexia/dificuldade, transtorno ou distúrbio de leitura; envolviam comorbidade como autismo, discalculia, distúrbio de 
linguagem, etc.; envolviam a dislexia adquirida; não envolviam a avaliação da leitura; e usavam como metodologia o estudo de caso ou revisão de literatura.

Após a aplicação dos critérios de inclusão e exclusão, e a retirada de trabalhos que se repetiam nas bases de publicação, foram selecionados 28 trabalhos, sendo 9 do PUBMED, 14 dos Periódicos da CAPES, 4 da BVS e uma publicação encontrada nas referências citadas nos trabalhos pesquisados, os quais encontravam-se em outros periódicos não alcançados pela pesquisa realizada (tabela1).

Tabela 1 - Fonte e total dos artigos encontrados para revisão de literatura

\begin{tabular}{l|c|c|c|c}
\hline \multirow{2}{*}{ Descritores } & \multicolumn{3}{|c}{ Fonte de pesquisa } \\
\cline { 2 - 5 } & $\begin{array}{c}\text { Periódicos } \\
\text { CAPES }\end{array}$ & $\begin{array}{c}\text { Biblioteca Vir- } \\
\text { tual de Saúde } \\
\text { (BVS) }\end{array}$ & PUBMED & $\begin{array}{c}\text { Seleção } \\
\text { manual }^{(1)}\end{array}$ \\
\hline Dislexia e funçóes executivas & 134 & 69 & 86 & 1 \\
Transtorno de leitura e funções executivas & 10 & 66 & 6 & - \\
Dificuldade de leitura e funçóes executivas & 4 & 14 & 19 & - \\
Deficiência de leitura e funçóes executivas & 55 & 66 & 10 & - \\
TOTAL & 204 & 215 & 121 & 1 \\
Selecionados após leitura dos resumos e artigos & 14 & 4 & 9 & 1 \\
Total de trabalhos selecionados = 28 & & & & \\
\hline
\end{tabular}

Fonte: elaboração própria.

(1) Encontrados em referência bibliográfica citada em trabalhos da área.

Foram encontradas algumas revisóes de literatura relacionadas com o tema proposto neste artigo. Um artigo sobre FE e aprendizagem (CORSO et al., 2013) o qual não aborda especificamente a leitura; uma metanálise que aborda a cognição em crianças com transtorno de leitura (KUDO; LUSSIER; SWANSON, 2015); e uma segunda meta-analise que avalia os testes usados para aferir as FE em crianças com dificuldade de leitura (BOOTH; BOYLE; KELLY, 2010). Nenhum, porém pretendia fazer um levantamento sistemático das pesquisas empíricas envolvendo as FE e a leitura em disléxicos.

\section{Resultados}

A primeira parte dos resultados apresenta uma caracterização geral do estudos, contendo os períodos de publicação, as temáticas estudadas e o público-alvo dos trabalhos selecionados. A segunda parte apresenta, quais os instrumentos mais usados para avaliar as FE e o que dizem os estudos sobre o impacto destas funçóes na leitura. A parte final dos resultado apresenta os estudos que realizaram intervenção nas $\mathrm{FE}$ em disléxicos e as consideraçóes gerais sobre o impacto das FE na dificuldade de leitura nas pessoas dislexia.

Neste trabalho será utilizado de forma generalizada o termo dislexia para se referir às dificuldades de leitura ou transtornos de leitura descritos anteriormente. 
Como a busca por publicações não limitou uma data de início, foram incluídos todos os trabalhos encontrados até junho de 2016. A primeira publicação encontrada é datada de 2000, e nos 10 primeiros anos a quantidade de publicaçóes foi discreta, tendo apenas 10 estudos sobre FE e dislexia. De 2011 até 2016 foram publicados 18 trabalhos, o que mostra um maior interesse dos estudiosos em entender de que forma as FE e a dislexia estão relacionadas.

Assim como os demais estudos envolvendo a dislexia, esta revisão confirma que a dificuldade de leitura está mais presente em meninos do que em meninas, porém poucos trabalhos compararam esta diferença em disléxicos. Altemeier, Abboth e Berninger (2008) identificaram que meninos com dislexia têm maior dificuldade em CI que meninas, mas não em RAS (Rapid Automatized Switch - alternância automática rápida). Este mesmo achado não foi encontrado em meninos do grupo controle sem dislexia.

Um estudo usou a metodologia longitudinal, avaliando crianças anualmente da pré-escola ao início da alfabetização, com o intuído de verificar os preditores da dislexia (THOMPSON et al., 2015). Um estudo apresentou os resultados obtidos ao longo de 12 anos de avaliação e intervenção de um Centro Multidisciplinar de Dificuldade de Aprendizagem na Universidade de Washington (BERNINGER et al., 2008). Quatro estudos realizaram intervenção, porém não especificamente em FE (LUO et al., 2013; HOROWITZ-KRAUS; BREZNITZ, 2014; HOROWITZ-KRAUS; TORO-SEREY; DIFRANCESCO, 2015; WALDA et al., 2014).

Vinte estudos investigaram as FE de forma geral, quatro pesquisaram as $\mathrm{FE}$ e a atenção (LIMA; AZONI; CIASCA, 2011, 2013; LIMA et al., 2012; BERNINGER et al., 2016), cinco estudaram especificamente a MT (SWANSON; SACHSE-LEE, 2001; JEFFRIES; EVERATT; 2004; BERNINGER et al., 2008; WANG; GATHERCOLE, 2013; LUO et al., 2013); um estudou a MT e o CI (BOOTH et al., 2014), três estudaram apenas o CI (SCHMID; LABUHN; HASSELHORN, 2011; BEXKENS; WILDENBERG; TIJMS, 2015; WANG; GATHERCOLE, 2015) e um estudou o controle inibitório e a FC (KAPOULA et al., 2010).

A maioria dos estudos envolveu crianças com idade média entre 9 e 11 anos. Um estudo investigou crianças com idade pré-escolar (THOMPSON et al., 2015), cinco investigaçóes envolveram adolescentes (BROSNAN et al., 2002; KAPOULA et al., 2010; HOROWITZ-KRAUS; BREZNITZ, 2014; HOROWITZ-KRAUS, 2014; HOROWITZKRAUS; TORO-SEREY; DIFRANCESCO, 2015), e um trabalho foi realizado também com adultos (BROSNAN et al., 2002). Com relação a metodologia, 94\% dos trabalhos utilizou grupo controle de leitores típicos para comparar o desempenho dos estudantes com dislexia, sendo a maioria deles pareados por idade.

\subsection{Memória de trabalho}

O teste mais utilizado, entre os estudos para avaliar a MT é o Digit Span ou Dígitos (BROSNAN et al., 2002; REITER; TUCHA; LANGE, 2005; SCHMID; LABUHN; HASSELHORN, 2011; LIMA; AZONI; CIASCA, 2013; CRUZ-RODRIGUES et al., 2014).

Inicialmente o déficit da MT não ficava claro, pois não se podia afirmar apenas com os resultados do Dígitos (BROSNAN et al., 2002). Conforme as pesquisas foram avançando, atualmente este teste é usado como uma das principais medidas para a MT fonológica, e 
pode ser considerado um preditor da dislexia (SWANSON; SACHSE-LEE, 2001; JEFFRIES; EVERATT, 2004; REITER; TUCA; LANGE, 2005; SCHMID; LABUHN; HASSELHORN, 2011), principalmente no sentido inverso, que exige reorganização de estímulo.

O instrumento utilizado para avaliar o componente visuoespacial da MT é o Blocos de Corsi ou Cubos de Corsi (CRUZ-RODRIGUES et al. (2014). Os estudos mostram que não há déficit no componente visuoespacial da MT em disléxicos (CRUZ-RODRIGUES et al., 2014; VARVARA et al., 2014), apenas quando associado ao CI (BOOTH et al., 2014). Segundo Brosnan et al. (2002), o CI e a MT não podem ser descritos de forma isolada, pois ambos são interdependentes e pertinentes para entender a dislexia.

Nos estudos de Swanson e Sachse-Lee (2001), Wang e Gathercole (2013) e Varvara et al. (2014) os disléxicos apresentaram déficit no executivo central. Berninger et al. (2008) colocam que déficits na MT podem explicar os problemas na decodificação, soletração e fluência em indivíduos com dislexia.

A partir destes achados, conclui-se que a MT tem um grande impacto sobre a leitura tanto de leitores típicos e disléxicos, podendo ser apontada como uma das áreas deficitárias a ser considerada na intervenção para a leitura, o qual justificaria uma intervenção envolvendo a estimulação deste componente.

\subsection{Controle INIBITório}

Brosnan et al. (2002), Kapoula et al. (2010), Lima, Azoni e Ciasca (2011, 2013) e Wang e Gathercole (2015) utilizando o teste Stroop, identificaram maior número de erros das crianças com dislexia, assim como a execução das tarefas sendo realizada em tempo maior em comparação ao grupo controle. Isso mostra a incapacidade dos disléxicos de inibirem o processamento da informação que precisa ser ignorada, ou seja, problemas com interferências (BROSNAN et al., 2002). Wang e Gathercole (2015) colocam que o déficit identificado no paradigma Stroop em disléxicos, não pode ser prontamente explicado em termos de diminuição do controle de interferência, justamente por estes estudantes serem mais lentos para decodificar e este pode ser o fator que interfere no CI. Lima, Azoni e Ciasca (2013) levantam a hipótese de um déficit de nomeação automática rápida (RAN) interferindo no $\mathrm{CI}$, o que justificaria o maior tempo para as respostas.

O CI, segundo Bexkens, Wildenberg e Tijms (2015), avaliado com o Simon Task, que identifica a capacidade de suprimir informaçóes que interferem na realização de tarefas, está relacionado à RAN, mas não ao déficit da RAN apresentado em disléxicos, pois a leitura envolve, além de RAN, também as capacidades fonológicas e de velocidade de processamento que se encontram em déficit na dificuldade de leitura.

Schmid, Labuhn e Hasselhorn (2011) tinham como objetivo verificar se há relação entre a performance da inibição, avaliada com o teste Stop Sign, e o processamento fonológico a curto prazo, avaliado com o teste Dígitos, em crianças com dislexia e leitores típicos. Os resultados não indicaram diferenças entre os grupos na resposta à inibição com o instrumento usado. Este resultado também foi encontrado no estudo de Bexkens, Wildenberg e Tijms (2015) mostrando que não houve nenhuma variância comum entre capacidade fonológica de curto 
prazo e a resposta à inibiçãao. A partir disso, é possível afirmar que o processamento fonológico não dificulta ou regula a resposta à inibição em tarefa não verbal.

No teste Go/no Go, o avaliando, diante de um grupo de estímulos, deve emitir ou suprimir uma reação. O CI é avaliado pelos erros de ação e as omissóes. Este teste também fornece dados sobre a atenção e a impulsividade (REPPOLD; TRENTINI; PEDRON, 2012). No estudo de Reiter, Tucha e Lange (2005) não houve diferença significativa entre o desempenho de disléxicos em comparação ao grupo controle com o uso do teste Go/no Go.

No estudo longitudinal realizado para verificar as contribuiçôes das FE para a alfabetização, Altemeier, Abbott e Berninger (2008) mostram que inibição e alternância automática rápida (RAS), ambos avaliados com o efeito Stroop, contribuem para a leitura e escrita em leitores típicos, e as mesma são necessárias para suprimir distratores, assim como estão ligadas à conexão entre letra e som. Esse resultado também foi encontrado no grupo de disléxicos. Já o estudo de Booth et al. (2014) mostrou que o CI tem pouco impacto como preditor da dificuldade de leitura, apesar do déficit apresentado em disléxicos.

A partir destes achados, conclui-se que ainda existem elementos contraditórios relacionados ao efeito do CI na leitura de pessoas com dislexia, uma vez que o tipo da prova, seja ela verbal ou não verbal, interfere na predição, assim como o mesmo teste pode ser utilizado para avaliar diferentes FE e isto dificultar o estudo do fenômeno em questão.

A sugestão para este dilema talvez seja a de se aplicar mais de um instrumento para avaliar o CI, utilizando atividades auditivas, visuais e verbais, podendo assim ampliar as possibilidades de análise deste componente.

\subsection{FleXIBILIDADE COGNITIVA}

Segundo Malloy-Diniz et al. (2010), os testes indicados para avaliação da FC são Wisconsin Card Sorting Test - WCST (Teste Wisconsin de Classificação de Cartas) e o Teste das Trilhas (parte B).

O WCST não se limita apenas à avaliação da FC, mas também outros componentes do funcionamento executivo geral, como atenção, MT, planejamento, insight, capacidade de abstração e processos de categorização (REPPOLD; TRENTINI; PEDRON, 2012). Dos estudos que utilizaram esse instrumento, Reiter, Tucha e Lange (2005), Menghini et al. (2010) e Varvara et al. (2014) apontaram que não houve diferença significativa entre o grupo controle e o grupo de disléxicos. Na pesquisa de Cruz-Rodrigues et al. (2014) o grupo de disléxicos utilizaram mais tempo e mais tentativas para completar a tarefa, porém a quantidade de erros no teste foi quase a mesma que o grupo controle. Os estudos de Helland e Asbjørnsen (2000) e Lima, Azoni e Ciasca (2013) mostraram, diferentemente dos três estudos mencionados anteriormente, que os disléxicos apresentaram maior número de tentativas e erros para encontrar, manter e alternar estratégias de resolução de problemas.

Horowitz-Kraus (2014) investigou adolescentes com dislexia em uma versão computadorizada do WCST, o MCST (Madri Card Sort Test), para identificar as FE prejudicadas. Foi usada o EEG (Eletroencefalograma) como metodologia para medir a atividade cerebral relacionada a realização do MCST. Os jovens com dislexia mostraram-se mais lentos e fizeram mais erros que 
os leitores hábeis. Os resultados mostraram que este rebaixamento não é causado por inflexibilidade cognitiva, mas por déficits nos componentes mais básicos das FE, como MT e atenção, ou também pode estar correlacionado com um quadro geral de lentidão de processamento.

Lima, Azoni e Ciasca (2011; 2013), Lima et al. (2012), Reiter, Tucha e Lange (2005) e Moura, Simóes e Pereira (2015), por meio do teste de trilhas B, verificaram que os disléxicos levaram mais tempo que o grupo controle para realizar a tarefa, e em Lima, Azoni e Ciasca (2013), também apresentou mais erros. Considerando a complexidade desta tarefa que envolve processar estímulos visuais e conhecer a sequência de letras e números, parece que para os disléxicos a capacidade de coordenar o tempo, velocidade e qualidade de processamento encontra-se alterado (LIMA et al., 2012). A pesquisa de Moura, Simóes e Pereira (2015) comparando o desempenho do Trilhas B com outros instrumentos (Torre de Londres $\bigotimes$ ToL; Trilhas A; o teste FAS; Código e Procurar Símbolos do WISC-III - WECHSLER, 2002), concluiu que a FC foi a função que apresentou maior indicativo preditor da dislexia do desenvolvimento.

Como foi apresentado na discussão sobre o CI, Kapoula et al. (2010) incluíram no Teste Stroop, uma quarta parte com o intuito de avaliar a FC. Foi identificado que os disléxicos realizaram mais erros e levaram mais tempo para concluir as quatro etapas do teste Stroop, o que demonstra que não há evidencias de um déficit extra na FC, além do já observado em CI e atenção alternada. Altemeier, Abbott e Berninger (2008) aplicaram o mesmo instrumento com a mesma finalidade e obteve resultado semelhante.

A avaliação da FC nestes estudos evidencia o quanto crianças com dislexia levam mais tempo para realizar atividades que necessitem de prontidão para a alternância, demonstrando uma sobrecarga mental não observada em leitores típicos, porém, de todos os componentes das FE, este é o que apresenta menor impacto na leitura isoladamente.

\subsection{FluênCia Verbal}

O Teste de Fluência Verbal FAS (FV) é considerado um instrumento complexo por contemplar vários componentes das FE, uma vez que para executá-lo o indivíduo precisa pensar e responder rapidamente, obedecer a uma determinada regra, prestar atenção, automonitorar e resgatar no léxico mental a informação que se enquadra no critério (produção) que pode ser, por exemplo: pensar no maior número de palavras que comece com a letra F, A ou $S$ (fonológico) ou palavras de uma determinada categoria como animais ou frutas (categoria semântica), no período de um minuto. Com a avaliação da fluência verbal é possível captar dados de várias funçóes e processos cognitivos complexos como: iniciativa, FC, velocidade de processamento, abstração e planejamento, a MT, a recuperação de palavras no léxico mental presente na linguagem e a aptidão verbal (MOURA, SIMÓES, PEREIRA, 2013).

Nove estudos que pretendiam avaliar as FE em crianças com dislexia ou dificuldade de leitura, utilizaram o Teste de Fluência Verbal. Brosnan et al. (2002), Reiter, Tucha e Lange (2005), Lima, Azoni e Ciasca (2013) verificaram que o grupo de disléxicos se desempenhou significativamente inferior na categoria fonológica do teste, diferente da categoria semântica onde a diferença foi inexpressiva. 
Já nos estudos de Cruz-Rodrigues et al. (2014), Varvara et al. (2014) e Moura, Simóes e Pereira (2015), os sujeitos apresentaram déficit nos dois subtipos do teste de fluência verbal, demonstrando que estas tarefas estão envolvidas em mecanismos cognitivos complexos como MT, automonitoramento e flexibilidade mental. Menghini et al. (2010) usaram apenas o teste de fluência verbal categorial, e também encontraram o mesmo déficit em disléxicos.

Berninger et al. (2016) relacionaram o desempenho no teste FAS com a linguagem oral, a leitura e a escrita em crianças e adolescentes com dislexia. $\mathrm{O}$ estudo concluiu que a fluência verbal e a inibição estão significativamente correlacionadas com toda a aprendizagem da linguagem auditiva e verbal, ou seja, são correlacionadas com as medidas cognitivas envolvidas no processo de tradução linguística. Estes achados apontam que os estudantes estão constantemente fazendo traduções através dos domínios da cognição e linguagem durante a aprendizagem acadêmica, e que tal tradução pode ser difícil durante o desenvolvimento da aprendizagem da linguagem em crianças com dificuldade de aprendizagem.

A avaliação da fluência verbal demonstra ser eficiente como parâmetro das FE, por usar a atenção sustentada, a MT, o CI e a FC, e em função dos déficits encontrados em disléxicos, permitem apontá-la como preditora da dislexia, principalmente com o achado de Horowitz-Kraus, Toro-Serey e Difrancesco (2015) que usaram o subteste fonológico do teste FAS em disléxicos, o qual apresentou déficit antes e melhora após a intervenção na leitura.

\subsection{FunÇóES EXECUTIVAS DE ALTO FUNCIONAMENTO}

As funções de planejamento (com o ToL), sequenciamento (com o Arranjo de Figuras do WAIS-R/WISC III) e organização (com o teste de cartas TBR - To be recalled), foram avaliadas por Brosnan et al. (2002) em 9 adultos com dislexia e não encontraram diferenças significativas comparando-se a respectivo grupo controle. $\mathrm{O}$ mesmo resultado foi encontrado no estudo de Reiter, Tucha e Lange (2005) com o uso dos testes ToL, Trilhas-B e Stroop em crianças com dislexia, para avaliar planejamento e resolução de problemas. O que foi observado é que os disléxicos vivenciam dificuldade em tarefas envolvendo controle e registro de tempo de processamento o que vem afetar a acurácia da resposta. As mediçóes de tempo de reação e resposta inicial demonstram-se preservadas, porém quando avaliados em tarefas mais complexas, o tempo de planejamento e elaboração de estratégias torna-se maior.

Da mesma forma Lima, Azoni e Ciasca (2011; 2013), Lima et al. (2012) e Moura, Simóes e Pereira (2015) usaram a ToL e não encontraram diferença significativa de desempenho entre os grupos de disléxicos e controle. Porém em Lima, Azoni e Ciasca (2013), apesar de não ser encontrada diferença de escore entre grupos por estatística convencional, quando usada medida estatística de magnitude de efeito, verificou-se que o grupo de disléxicos manifestou déficit nesta tarefa.

Outros testes utilizados para avaliar planejamento e resolução de problemas são aqueles que envolvem a classificação de cartas, como o WCST (HELLAND; ASBJØRNSEN, 2000; MENGHINI et al., 2010), também utilizado para avaliar a FC (LIMA; AZONI; CIASCA, 2013).

Helland e Asbjørnsen (2000) quando utilizaram o WCST verificaram que a capacidade de gerar regras e seguir regras para a solução de problemas estava afetado em disléxicos. Menghini et al. (2010) usando o mesmo teste obtiveram resultado quase semelhante ao grupo 
controle, ou seja, sem um déficit significativo. Como também utilizaram o teste de Fluência Verbal (FAS) o qual mostrou-se rebaixado em disléxicos, é possível afirmar que existem falhas no planejamento, monitoramento e revisão durante a resolução de problemas.

$\mathrm{O}$ que pode se concluir a partir dos estudos, é que as FE de alto funcionamento, por estarem diretamente relacionadas com a inteligência, e a inteligência não estar prejudicada em disléxicos, é esperado que não haja um grande comprometimento nesta área. O que se observa é que quando se utiliza um instrumento que solicita a utilização de processamento que envolve a área fonológica, existe um déficit nos indivíduos com dislexia principalmente em tempo de realização.

O fato das FE se sobreporem durante a execução de uma tarefa se torna o maior desafio na análise de áreas isoladas e específicas, uma das possibilidades é a realização de mais de um instrumento que avalie a mesma área e com isso, verificar aquele que mais fortemente se correlaciona com determinada função e a dislexia.

\subsection{Intervenção e contribuições para a remediação}

Um treinamento computadorizado para estimulação da MT foi realizado por Luo et al. (2013) em crianças com dislexia o qual demonstrou melhora nas habilidades de leitura. $\mathrm{O}$ treino teve efeito positivo para tarefas de rima e fluência de leitura. Swanson e Sachse-Lee (2001) apontam que a intervenção na MT em leitores típicos e disléxicos, melhora a MT, pois durante sua pesquisa, foram realizadas reaplicaçóes que permitiram aos alunos corrigirem alguns erros e demonstrarem desempenho melhor que o anterior.

Horowitz-Kraus, Toro-Serey e Difrancesco (2015) realizaram uma intervenção utilizando um programa de aceleração da leitura computadorizado que resultou em melhora de todas as FE e na leitura. Os resultados foram comprovados por meio de ativação cerebral mensurado pelo EEG (Eletroencefalograma). Disléxicos foram mais lentos em FE, e após a intervenção melhoraram em atenção (tempo e acurácia), inibição, velocidade de processamento, flexibilidade e FE em geral avaliadas pelo WSCT.

$\mathrm{O}$ único estudo que considera que uma intervenção das FE não melhora o desempenho na leitura é o de Walda et al. (2014). Os pesquisadores realizaram um estudo longitudinal para investigar se a leitura em crianças com dislexia é afetada pelas FE. Os resultados mostraram que as FE são preditivas da dislexia, porém não ao progresso na leitura e ortografia durante o processo de remediação. Foi observado um progresso nas FE durante a remediação (mesmo sem avaliar formalmente), o que propóe a hipótese de que o déficit nas $\mathrm{FE}$ e na leitura são causados pelos mesmo problemas (déficit cognitivo e fonológico). O estudo mostrou que não é possível usar o resultado nas FE para prever a evolução futura na leitura e na escrita, e nem que um treinamento nas FE pode afetar o desempenho na leitura, porém não foi aplicado pós-teste para comprovar estes resultados.

Sabe-se que a intervenção fonológica é eficiente como remediadora da dislexia, porém o que se observa é que a intervenção envolvendo as FE pode melhorar não somente as FE, mas também a leitura e sua compreensão. Talvez o que seja eficiente não é intervir diretamente para melhorar as FE, mas incluir tarefas que façam uso das FE para a aprendizagem da leitura. 


\subsection{FUNÇÓES EXECUTIVAS COMO PREDITORAS DA DISLEXIA}

Todos os estudos selecionados para esta revisão apontaram déficits nas FE em disléxicos, seja quando avaliadas como um componente único seja quando seus componentes foram avaliados isoladamente, porém, estes déficits podem estar presentes em alguns disléxicos, mas náo em todos, ou ainda o déficit náo ser homogêneo, ou seja, nas mesmas áreas para cada um. Isso se dá pelo fato da dislexia apresentar déficit multifatorial, e sua disfunção não ser limitada apenas às áreas linguísticas do cérebro, sendo que diferentes indivíduos podem apresentar deficiências neuropsicológicas e dificuldades cognitivas distintas nas avaliaçôes, porém no conjunto, manifestarem habilidade de leitura igualmente pobre (MENGHINI et al., 2010; VARVARA et al., 2014). Isso é possível verificar no estudo de Menghini et al. (2010) que avaliou 60 disléxicos e identificou que apenas 18,3\% deles apresentou déficit fonológico exclusivamente, e que $76,6 \%$ mostraram outros déficits além do fonológico, sendo $38,2 \%$ deles com déficit nas FE. Com isso é possível afirmar que vários disléxicos apresentam baixo desempenho nas $\mathrm{FE}$, porém não todos. Outro aspecto a ser considerado é o fato de que as $\mathrm{FE}$ rebaixadas não serem suficientes para afirmar se são preditores ou náo da dislexia, pois depende do quanto estas funçóes contribuem com a alfabetização (ALTEMEIER; ABBOTT; BERNINGER, 2008).

Thompson et al. (2015) acompanharam crianças pré-escolares com histórico de risco familiar para dislexia em um estudo longitudinal testando também as FE. Os autores concluíram que as FE são preditoras adicionais da dificuldade de leitura na pré-escola, juntamente com o conhecimento de letras, a consciência fonológica e a nomeação automática rápida, porém somente com a introduçáo do ensino da leitura e escrita é possível confirmar os riscos para a dislexia. As capacidades de linguagem e as habilidades motoras foram os preditores mais significativos. Apesar de rebaixadas minimamente nas crianças em risco para a dislexia, as FE não tiveram impacto como preditoras, porém têm o papel de fornecer compensação para a baixa disposição à leitura em disléxicos.

O processamento da leitura é facilitado quando se inibem os distratores em um nível mais global, pois do contrário, os mesmos podem atrasar todo o processo. O estudo de Brosnan et al. (2002) mostrou que existe uma falha no processamento inibitório no nível logográfico (da palavra) que afeta a leitura processada via correspondência grafema-fonema em disléxicos. Existe uma tendência a afirmar que a dificuldade de leitura (decodificaçáo grafema-fonema) na dislexia pode ser vista como um atraso assimétrico no processamento afetado pelo CI e MT ineficientes. A dificuldade de inibiçâo de estímulos (CI) foi apontada como preditora da dislexia nos estudos de Altemeier, Abbott e Berninger (2008), Varvara et al. (2014), Booth et al. (2014) e Wang e Gathercole (2015), e o déficit em MT nos estudos de Swanson e Sachse-Lee (2001), Jeffries e Everatt (2004), e Horowitz-Kraus (2014). Alguns estudos também apontaram a velocidade de processamento como um dos componentes que prediz a dislexia, juntamente com a FC (REITER; TUCHA; LANGE, 2005; ALTEMEIER; ABBOTT; BERNINGER, 2008; BEXKENS; WILDENBERG; TIJMS, 2015).

Em leitores típicos, quando a decodificaçáo e o reconhecimento de palavras familiares foi aprendido, as FE garantem que este processo ocorra em tempo, com acurácia (ALTEMEIER; ABBOTT; BERNINGER, 2008). Em muitas testagens, disléxicos demonstraram precisar de mais tempo para pensar e planejar, o que afeta diretamente a produtividade na leitura (REITER; TUCHA; LANGE, 2005; BROSNAN et al., 2002; KAPOULA et al. 2010; LIMA; ANZONE; 
CIASCA, 2011; 2013; WANG; GATHERCOLE, 2015). Segundo Berninger et al. (2008), as FE predizem a habilidade de integrar a leitura e a escrita nas crianças com dislexia.

As FE de alto funcionamento de planejamento, monitoramento e revisão para a resolução de problemas também são apontadas como preditores da compreensão leitora por Menghini et al. (2010) e Altemeier, Abbott e Berninger (2008), pois as FE governam e integram informações para a resolução de problemas, inclusive na leitura.

Diante da complexidade presente no quadro da dislexia, não há consenso entre os estudiosos quanto ao efeito preditor das FE de forma isolada, na dislexia do desenvolvimento. Existe rebaixamento de algumas FE nos disléxicos, como a MT e o CI, porém esse rebaixamento não é homogêneo em todos os indivíduos pesquisados, portanto não seria preditor. Além disso os déficits apontados não permitem afirmar que a dislexia seja uma síndrome desexecutiva, pois não atingem um nível de disfunção do lobo pré-frontal, mesmo assim não se pode negar a importância das funções executivas para a leitura e também para a compensação do seu déficit em disléxicos (REITER; TUCHA; LANGE, 2005).

\section{Conclusáo}

Os estudos selecionados para esta revisão não avaliam as FE da mesma forma. Alguns avaliam as FE como um componente global, o qual demonstra pouca diferença comparando os disléxicos a leitores típicos, outros avaliam os componentes principais das $\mathrm{FE}$, que são o $\mathrm{CI}$, a FC e a MT, ou ainda outros que avaliavam também os subcomponentes, como por exemplo na MT.

Foram usados diferentes instrumentos, abrindo a possibilidade para os estudos divergirem em relação aos resultados. A impureza dos testes que avaliam as FE também é outro elemento que interfere nas conclusóes, pois em algumas situaçóes, o mesmo teste pode avaliar mais de uma função, dificultando que se mensure precisamente cada componente.

Os componentes das FE estão associados e integrados, fazendo com que a MT afete a FC, assim como o CI afete a MT, e assim por diante. O que fica evidente é o impacto que as FE têm nas estratégias de leitura que envolvem a manipulação da informação, o tempo de resposta, a capacidade de inibição de estímulos distratores, assim como a alternância entre diferentes elementos com significados distintos.

A MT apresentou déficit em vários estudos quando avaliada como componente integral, e quando avaliados os seus subcomponentes isoladamente os estudos não tiveram consonância. Ainda assim, é possível afirmar que o resultado na leitura depende da performance da MT.

No componente CI alguns estudos identificaram maior número de erros das crianças com dislexia, assim como a execução das tarefas em tempo maior em comparação aos grupos de controle, como se os disléxicos tivessem uma tendência em se distrair, enquanto que em outros estudos não houve diferença significativa.

Com relação à FC houve distinções significativas entre os estudos em função do instrumento utilizado. O Teste de Trilhas-B demonstra ser o teste mais sensível para identificar o déficit deste componente, que se mostrou em déficit nos sujeitos com dislexia. 
Os instrumentos usados para avaliar a fluência verbal mostraram-se eficientes, porém os resultados entre os disléxicos e os leitores típicos tiveram diferença significativa.

Os trabalhos com esta temática são escassos. A maioria dos estudos envolveu população estrangeira (estadunidenses, alemães, italianos, ingleses, holandeses, israelenses e portugueses), apenas quatro trabalhos foram realizados com a população brasileira. Essas diferenças de resultados entre os estudos podem ser em decorrência da pouca quantidade de pesquisa; dos tipos de instrumentos utilizados; da quantidade de instrumentos utilizados para avaliar cada componente das FE e da análise estatística usada para tratar os dados, o que mostra a necessidade de ampliar e aprofundar essa temática de forma atualizada e sistemática, com instrumentos validados e padronizados para a população brasileira.

Poucos estudos possuíam grupo controle pareado pelo nível de leitura das crianças disléxicas. A prática das pesquisas é comparar as crianças com dislexia com crianças da mesma faixa etária, porém sabe-se que comparar com crianças com o mesmo nível de leitura pode enriquecer os achados da pesquisa e contribuir para identificar como o processamento cognitivo semelhante ao dos disléxicos se desempenha nas avaliaçóes.

A maioria dos estudos, com exceção do de Walda et al. (2014), sugerem o desenvolvimento de programas de intervenção para pessoas com dislexia, envolvendo as FE. Walda et al. (2014) consideram que a dislexia do desenvolvimento é caracterizada exclusivamente pelo déficit fonológico, e que um programa de intervenção que tem como foco a estimulação das FE teria um benefício menos importante se comparado a outras intervençóes voltadas para a leitura, a decodificação, a ortografia e a consciência fonológica. O estudo de Walda et al. (2014) não comparou os resultados com grupo controle, o que demonstra a necessidade de novos estudos sobre esta temática.

A partir desta revisão, observa-se que o estudo das FE em disléxicos está em pleno desenvolvimento. Nos últimos 5 anos houve um interesse maior de se entender esta temática, inclusive no Brasil, porém ainda há muito que se conhecer, principalmente sobre o efeito de intervençóes envolvendo as FE. Faz-se necessário apresentar os benefícios que estas intervençóes podem trazer para a prática da sala de aula, mostrando evidências do ensino que tem como pano de fundo o uso de estratégias envolvendo as FE no favorecimento da aprendizagem das crianças com dificuldade de leitura.

\section{REFERÊNCIAS}

ALTEMEIER, L. E.; ABBOTT, R.D.; BERNINGER, V.W. Executive functions for reading and writing in typical literacy development and dyslexia. Journal of clinical and experimental neuropsychology, Victoria, BC, Canada, v.30, n.5, p.588-606, 2008.

ALVES, L.M. et al. Introdução à dislexia do desenvolvimento. In: ALVES, L. M.; MOUSINHO, R.; CAPELLINI, S.A. (Org.). Dislexia: novos temas, novas perspectivas. Rio de Janeiro: Wak, 2011. p.21-40.

AMERICAN PSYCHIATRIC ASSOCIATION (APA). Manual diagnóstico e estatístico de transtornos mentais: DSM-5. 5. ed. Porto Alegre: Artmed, 2014.

BADDELEY, A. D. Working memory: looking back and looking forward. Nature reviews neuroscience, London, v.4, n.10, p.829-839, 2003. 
BERNINGER, V. et al. Relationships of attention and executive functions to oral language, reading, and writing skills and systems in middle childhood and early adolescence. Journal of learning disabilities, Riverside, USA, p.1-15, jan. 2016.

BERNINGER, V.W. et al. Multidisciplinary approach to understanding developmental dyslexia within working-memory architecture: Genotypes, phenotypes, brain, and instruction. Developmental neuropsychology, United Kingdom v.33, n.6, p.707-744, 2008.

BEXKENS, A.; WILDENBERG, W.P.M.; TIJMS, J. Rapid automatized naming in children with dyslexia: is inhibitory control involved? Dyslexia, Anglia, UK, v.21, n. 3, p.212-234, 2015.

BOOTH, J.N. et al. The relationship between inhibition and working memory in predicting children's reading difficulties. Journal of research in reading, United Kingdom, v.37, n.1, p.84-101, 2014.

BOOTH, J.N.; BOYLE, J.M.E.; KELLY, S.W. Do tasks make a difference? Accounting for heterogeneity of performance of children with reading difficulties on tasks of executive function: Findings from a metaanalysis. British Journal of Developmental Psychology, Changai, v.28, n.1, p.133-176, 2010.

BOVO, E.B.P. et al. Relações entre as funçôes executivas, fluência e compreensão leitora em escolares com dificuldades de aprendizagem. Revista Psicopedagogia, v.33, n.102, p.272-282, 2016.

BROSNAN, M. et al. Executive functioning in adults and children with developmental dyslexia. Neuropsychologia, Dallas, v.40, n.12, p.2144-2155, 2002.

CAIN, K. Reading development and difficulties. British Psychological Society and Blackwell United Kingdom, Publishing. 2010.

CAPELLINI, S.A. et al. Desempenho de escolares bons leitores, com dislexia e com transtorno do déficit de atenção e hiperatividade em nomeação automática rápida. Revista da Sociedade Brasileira de Fonoaudiologia, Campinas, v.12, n.2, p.114-119, 2007.

CARTWRIGHT, K.B. Executive skills and reading comprehension: A guide for educators. New York: Guilford Publications, 2015.

CORSO, H.V. et al. Metacognição e funçóes executivas: relaçóes entre os conceitos e implicaçóes para a aprendizagem. Psicologia: Teoria e Prática, São Paulo, v.29, n.1, p.21-29, 2013.

CRUZ-RODRIGUES, C. et al. Neuropsychological characteristics of dyslexic children. Psicologia: Reflexão e Crítica, Porto Alegre, v.27, n.3, p.539-546, 2014.

CUNHA, V.L.O. et al. Desempenho de escolares com transtorno de déficit de atenção e hiperatividade em tarefas metalinguísticas e de leitura. Revista CEFAC, São Paulo, p.1-11, 2013.

DIAMOND, A. Executive functions. Annual Review of Psychology. Princeton, NJ, v.64, p.135-168, 2013.

ELLIS, A.W. Leitura, escrita e dislexia: uma abordagem cognitiva. 2.ed. Porto Alegre: Artes Médicas, 1995.

GILBERT, S.; BURGESS, P.W. Executive function. Current Biology, United Kingdom, v.18, n.3, p.110-114, 2008.

HELLAND, T.; ASBJØRNSEN, A. Executive functions in dyslexia. Child Neuropsychology, United Kingdom, v.6, n.1, p.37-48, 2000.

HOROWITZ-KRAUS, T. Pinpointing the deficit in executive functions in adolescents with dyslexia performing the Wisconsin Card Sorting Test An ERP Study. Journal of Learning Disabilities, Riverside, USA, v.47, n.3, p.208-223, 2014. 
HOROWITZ-KRAUS, T.; BREZNITZ, Z. Can reading rate acceleration improve error monitoring and cognitive abilities underlying reading in adolescents with reading difficulties and in typical readers? Brain Research, Boston, v.1544, p.1-14, 2014.

HOROWITZ-KRAUS, T.; TORO-SEREY, C.; DIFRANCESCO, M. Increased resting-state functional connectivity in the cingulo-opercular cognitive-control network after intervention in children with reading difficulties. PloSone, San Francisco, California, US, v.10, n.7, p.e0133762, 2015.

JEFFRIES, S.; EVERATT, J. Working memory: its role in dyslexia and other specific learning difficulties. Dyslexia, Anglia, UK, v.10, n.3, p.196-214, 2004.

KAPOULA, Z. et al. Poor Stroop performances in 15-year-old dyslexic teenagers. Experimental Brain Research, v.203, n.2, p.419-425, 2010.

KUDO, M.F.; LUSSIER, C.M.; SWANSON, H.L. Reading disabilities in children: A selective metaanalysis of the cognitive literature. Research in Developmental Disabilities, United Kingdom, v.40, p.51-62, 2015.

LEZAK, M.D. The problem of assessing executive functions. International Journal of Psychology, United Kingdom, v.17, n.1-4, p.281-297, 1982.

LIMA, R.F. de et al. Atención sostenida visual y funciones ejecutivas en niños con dislexia de desarrollo. Anales de Psicología, Murcia España, v.28, n.1, p.66-70, 2012.

LIMA, R. F. de; AZONI, C. A. S.; CIASCA, S. M. Atenção e funções executivas em crianças com dislexia do desenvolvimento. Psicologia em Pesquisa, Juíz de Fora, v.7, n.2, p.208-219, 2013.

LIMA, R.F. de; AZONI, C.A.S.; CIASCA, S.M. Attentional performance and executive functions in children with learning difficulties. Psicologia: Reflexão e Crítica, Porto Alegre, v.24, n.4, p.685-691, 2011.

LIMA, R.F. de; TRAVAINI, P.P.; CIASCA, S.M. Amostra de desempenho de estudantes do ensino fundamental em testes de atenção e funções executivas. Revista Psicopedagogia, v.26, n.80, p.188-199, 2009.

LUO, Y. et al. Working-memory training improves developmental dyslexia in Chinese children. Neural Regeneration Research, Hong Kong, v.8, n.5, p.452, 2013.

MALLOY-DINIZ, L.F. et al. Exame das funçóes executivas. In: MALLOY-DINIZ, L.F. et al. (Org.). Avaliação neuropsicológica. Porto Alegre: Artmed, 2010. p.94-113.

MALLOY-DINIZ, L. F. et al. Neuropsicologia das funções executivas e da atenção. In: FUENTES, D. et al. (Org.). Neuropsicologia: teoria e prática. 2. ed. Porto Alegre: Artmed, 2014. p.115-138.

MENGHINI, D. et al. Different underlying neurocognitive deficits in developmental dyslexia: a comparative study. Neuropsychologia, United Kingdom, v.48, n.4, p.863-872, 2010.

MIYAKE, A. et al. The unity and diversity of executive functions and their contributions to complex "frontal lobe" tasks: A latent variable analysis. Cognitive Psychology, United Kingdom, v.41, n.1, p.49100, 2000.

MOURA, O.; SIMÓES, M.R.; PEREIRA, M. Executive functioning in children with developmental dyslexia. The Clinical Neuropsychologist, United Kingdom, v.28, n. sup1, p.20-41, 2015.

MOURA, O.; SIMÓES, M.R.; PEREIRA, M. Fluência verbal semântica e fonêmica em crianças: funçóes cognitivas e análise temporal. Avaliaçáo Psicológica, Itatiba, v.12, n.2, p.167-177, 2013. 
PUREZA, J. da et al. Funçôes executivas: fundamentos teóricos e implicaçôes clínicas e educacionais. In: MOUSINHO, R.; ALVES, L.M.; CAPELLINI, S.A. (Org.). Dislexia: novos temas, novas perspectivas. Rio de Janeiro: Wak, 2015. v.3. p.103-122.

REITER, A.; TUCHA, O.; LANGE, K.W. Executive functions in children with dyslexia. Dyslexia, Anglia, UK, v.11, n.2, p.116-131, 2005.

REPPOLD, C.T.; TRENTINI, C.M.; PEDRON, A. C. Regendo a orquestra: funçôes executivas. In: HUTZ, C.S. (Org.). Avanços em avaliação psicológica e neuropsicológica de crianças e adolescentes - II. São Paulo: Casa do Psicólogo, 2012. p.143-167.

SCHMID, J.M.; LABUHN, A.S.; HASSELHORN, M. Response Inhibition and its Relationship to Phonological Processing in Children with and without Dyslexia. International Journal of Disability, Development and Education, United Kingdom, v.58, n.1, p.19-32, 2011.

SEABRA, A.G. et al. Inteligência e funçôes executivas: avanços e desafios para a avaliação neuropsicológica. São Paulo: Memnon, 2014.

SNOWLING, M.J. Dislexia. Santos: Livraria Santos Editora, 2. ed. 2004.

SWANSON, H.L.; SACHSE-LEE, C. A subgroup analysis of working memory in children with reading disabilities domain-general or domain-specific deficiency? Journal of learning disabilities, Riverside, USA, v.34, n.3, p.249-263, 2001.

THOMPSON, P.A. et al. Developmental dyslexia: predicting individual risk. Journal of Child Psychology and Psychiatry, New Jersey, v.56, n.9, p.976-987, 2015.

VARVARA, P. et al. Executive functions in developmental dyslexia. Frontiers in Human Neuroscience, Lausanne Switzerland, v.8, p.120, 2014.

VELLUTINO, F.R., FLETCHER, J.M. Dislexia do Desenvolvimento. In: SNOWLING, M. J.; HULME, C. (Org.). A ciência da leitura. Porto Alegre: Penso, 2013.

WALDA, S.A. et al. Progress in reading and spelling of dyslexic children is not affected by executive functioning. Research in Developmental Disabilities, United Kingdom, v.35, n.12, p.3431-3454, 2014.

WANG, S.; GATHERCOLE, S.E. Interference control in children with reading difficulties. Child Neuropsychology, United Kingdom, v.21, n.4, p.418-431, 2015.

WANG, S.; GATHERCOLE, S.E. Working memory deficits in children with reading difficulties: Memory span and dual task coordination. Journal of Experimental Child Psychology, United Kingdom, v.115, n.1, p.188-197, 2013.

WECHSLER, D. WISC-III: escala de inteligência para crianças: manual. 3. ed. Adaptação e padronização brasileira de Vera Lúcia Marques de Figueiredo. São Paulo: Casa do Psicólogo, 2002.

Recebido em: 14/04/2017

Reformulado em: 31/08/2017

Aprovado em: 11/09/2017 